
\title{
25 Research Square \\ Clinical Correlates of Early IgG Response in Patients with Mild COVID-19
}

\section{Asa Kessler}

Hadassah Medical Center

Ariel Kenig ( $\boldsymbol{\nabla}$ kenig.ariel@gmail.com )

Hadassah Medical Center https://orcid.org/0000-0003-2310-6118

Henny Azmanov

Hadassah Medical Center

\section{Ram Gelman}

Hadassah Medical Center

Noa Hurvitz

Hadassah Medical Center

Itay Perets

Hadassah Medical Center

Esther Oiknine-Djian

Hadassah Medical Center

\section{Tali Bdolah-Abram}

Hebrew University Hadassah Medical School

\section{Orly Zelig}

Hadassah Medical Center

\section{Zeev Rotstein}

Hadassah Medical Center

\section{Yaron Ilan}

Hadassah Medical Center

\section{Dana G. Wolf}

Hadassah Medical Center

\section{Research article}

Keywords: SARS-CoV-2, COVID-19, Serology, SARS-CoV-2-Specific Antibodies, Convalescent plasma

Posted Date: August 26th, 2020

DOI: https://doi.org/10.21203/rs.3.rs-56853/v1 
License: (c) (i) This work is licensed under a Creative Commons Attribution 4.0 International License. Read Full License 


\section{Abstract}

Background: We determined the temporal pattern of early SARS-CoV-2 IgG response in patients with mild COVID-19, and sought to identify predictive clinical and laboratory features.

Methods: Serum samples were prospectively obtained from 111 convalescent COVID-19 patients, staying in dedicated Isolation-hotels, and tested for the presence of SARS-CoV-2 IgG by anti-S1 protein ELISA.

Results: SARS-CoV-2 IgG was detected in 78 (70.3\%) patients tested within the first month from diagnosis. While highly variable between patients, the rate of antibody detection generally increased with time, from $47.1 \%$ to $93.8 \%$ at the first and fourth weeks from diagnosis, respectively, with the largest shift observed between the second and third week. Notably, the presence of more profound symptoms at presentation, namely, fever and chills, positively and independently correlated with early antibody response. IgG-positive patients had higher ferritin levels $(p=0.039)$. Older age $(p<0.001)$ and increased CRP levels $(p=0.001)$ were associated with higher SARS-CoV-2 IgG levels.

Conclusions: The identified temporal pattern along with the correlation between inflammation-related clinical and laboratory parameters and early IgG response in patients with mild COVID-19, could provide a basis for better prediction and understating of the immune response to SARS-CoV-2, and inform therapeutic donor-plasma selection.

\section{Background}

Since its emergence in Wuhan, China, in December 2019, the novel severe acute respiratory syndrome coronavirus-2 (SARS-CoV-2), identified as the etiological agent of coronavirus disease-19 (COVID-19), has caused a rapidly spreading global pandemic associated with significant morbidity and mortality and farreaching economic and social implications $(1,2)$. By early June 2020, more than seven million confirmed cases and more than 400,000 SARS-CoV-2-related deaths have been reported worldwide. While the majority of infected patients are asymptomatic or present with mild disease, severe disease associated with acute respiratory distress syndrome (ARDS) and multi-organ failure complicate 5-20\% of COVID-19 cases (2-4). The diagnosis of active SARS-CoV-2 infection is based on the detection of viral RNA in nasopharyngeal swab sample by reverse transcription (RT)-real time polymerase chain reaction (PCR) (5). This assay, requiring initial viral inactivation step, skilled laboratory personnel, special laboratory facilities, and large volumes of RNA extraction and RT-PCR kits along with automated liquid handlers (to address the requirement for high throughput performance during the pandemic), has been complicated by diagnostic bottlenecks and reagents' shortage.

SARS-CoV-2 antibody detection assays are currently developed at a rapid pace. Serological studies should enhance the understanding of the immune response to SARS-CoV-2 (6), allow the diagnosis of recent or past infection (when RT-PCR was not performed or was false-negative) $(7,8)$, support population-based studies and public-health strategies, and inform vaccine development. Additionally, 
SARS-CoV-2 IgG detection allows for the initial screening of immune-donor plasma - recently shown to be a promising therapeutic approach in critically ill COVID-19 patients (9).

In Israel, the first cases of SARS-CoV-2 infection were confirmed in mid-February, and were followed by accelerated transmission during late-March through April - due to travel-associated importations, large holiday gatherings, and introductions into the densely populated orthodox-religious neighborhoods. During this period, recovering hospitalized COVID-19 patients with mild disease were routinely discharged into dedicated isolation hotels. The common location of these patients during the early convalescence phase presented us with an opportunity to determine the pattern and clinical correlates of early SARSCoV-2 IgG response in patients with mild disease - constituting the majority of COVID-19 patients.

\section{Patients And Methods}

\section{Study design and patient population.}

Included in the study were 111 adult patients who had been hospitalized with mild COVID-19, and were subsequently staying in dedicated isolation hotels in Jerusalem until recovery. The vast majority of patients did not require oxygen support (and none received noninvasive or mechanical ventilation) and no patient was admitted to the intensive care unit. Therefore, the patients were retrospectively defined as representing mild COVID-19 cases. The hospitalization in itself did not preclude the definition of mild disease as at this stage of the pandemic in Israel, all patients were initially hospitalized.

The time from diagnosis was defined as the time from the first recorded positive nasopharyngeal swab. Serum samples and concurrently obtained nasopharyngeal swab samples were prospectively collected once per each patient during April 2020 and analyzed for the presence of SARS-CoV-2 IgG and RNA, respectively. Blood samples were also analyzed for blood count, C-reactive protein (CRP) and ferritin levels.

The demographic and clinical data were collected from the medical files, and analyzed retrospectively (see Table 1 for analyzed parameters). The study was approved by the Institutional review board and was performed according to the Human-Experimentation Guidelines of the Israeli Ministry of Health. All patients provided an informed consent.

\section{SARS-CoV-2 IgG and RNA detection.}

Enzyme-linked immunosorbent assay (ELISA) for SARS-CoV-2 IgG (EUROIMMUN Corp., Germany), measuring IgG against the viral spike protein $\mathrm{S} 1$, was used in accordance with the manufacturer's instructions. Ratios (sample/calibrator readings at $450 \mathrm{nM}$ ) of $<0.8,0.8-1.1$, and $>1.1$ were determined as negative, intermediate, and positive IgG results, respectively. Antibody levels in this assay did not exceed

10.0 , therefore, based on the observed range of antibody levels (semi-quantitatively defined by the rations), a cutoff ratio of 5.0 was chosen to distinguish between high and low antibody levels. 
Nasopharyngeal swab samples were collected in $2 \mathrm{ml}$ Viral Transport Medium and mixed 1:1 with a $2 \mathrm{X}$ concentrated lysis buffer (DNA/RNA Shield; Zymo Research). Viral RNA was extracted using the QIAsymphony DSP Virus/Pathogen kit on a QIAsymphony platform (Qiagen) in accordance with the manufacturer's specifications. RNA was eluted into $60 \mathrm{ml}$, and $10 \mathrm{ml}$ of the eluted RNA was used for a 30 ml RT-PCR reaction, using Real-Time Fluorescent RT-PCR kit with primers and probe targeted to the SARSCoV-2 ORF1ab gene (BGI).

\section{Statistical analysis.}

The Chi-square test and Fisher's exact test were used to determine the association between two categorical variables. Comparison of continuous variables between two independent groups was carried out using two-sample t-test for normally distributed data and Mann-Whitney non-parametric test for nonnormally distributed data. In order to simultaneously assess the effect of several variables on the dependent variable (i.e., positive/negative serology), while correcting for confounders, the multivariate logistic regression model was applied using the stepwise, forward, likelihood ratio method. All tests applied were two-tailed, and a p-value of 0.05 or less was considered statistically significant. Statistical analysis was performed using IBM SPSS Statistics, version 25.

\section{Results}

\section{Temporal pattern of IgG response in convalescent COVID-19 patients.}

Within a month from the first positive nasopharyngeal swab, SARS-CoV-2 IgG was detected in 78 (70.3\%) of 111 recovering COVID-19 patients, 6 (5.4\%) patients had intermediate IgG results, and $27(24.3 \%)$ patients were found to be negative for SARS-CoV-2 IgG

Figure 1 presents the rate of SARS-CoV-2 IgG detection by the time from diagnosis, ranging from $47.1 \%$ to $93.8 \%$ among patients tested during the first and fourth week form diagnosis, respectively $(p=0.006)$. Five of six intermediate IgG results were obtained in patients tested during the second week (days 8-14), with the greatest antibody detection shift noticed between the second and third week post diagnosis. The mean time from diagnosis was significantly longer for patients with positive SARS-CoV-2 IgG $(15.6 \pm 6.0$ vs. $11.0 \pm 4.9$ days in IgG negative patients; $p<0.001)$, as also demonstrated for the mean time from symptoms onset $(21.3 \pm 8.9$ vs. $13.6 \pm 8.3$ days; $p<0.001)$. The longest time from symptoms onset during which a patient remained IgG negative was 44 days, while the shortest time from symptoms onset to $\lg G$ positivity was 8 days.

\section{Comparison of demographic, clinical, and laboratory features between IgG-positive and IgG-negative patients.}

Comparison of IgG-positive and IgG-negative patients revealed no significant differences in demographic characteristics and in the presence of underlying medical conditions (Table 1). Smoking and hypertension were the most common comorbidities in both groups. Only three patients had underlying 
immunosuppression (kidney transplantation, multiple sclerosis, and chronic inflammatory demyelinating polyneuropathy).

Table 1: Comparison of demographic and clinical characteristics between SARS-CoV-2 IgG-positive and IgGnegative patients 
$\mathrm{N}(\%)$ of patients ${ }^{\mathrm{a}}$

All Patients Antibody-positive Antibody-negative

Characteristic

$(\mathrm{n}=111)$

$(\mathrm{n}=78)$

$(n=27)$

$P$ value

\section{Age group}

$<30$

$30-39$

$36(32.4)$

$22(28.2)$

9 (33.3)

0.63

$40-49$

21 (18.9)

15 (19.2)

$6(22.2)$

$50-59$

$12(10.8)$

8 (10.3)

4 (14.8)

$60-69$

$16(14.4)$

12 (15.4)

3 (11.1)

$70-79$

18 (16.2)

15 (19.2)

3 (11.1)

$\geq 80$

7 (6.3)

6 (7.7)

1 (3.7)

$1(0.9)$

$0(0.0)$

1 (3.7)

\section{Sex}

\section{Male}

Female

Underlying medical conditions

Any

Smoking

Diabetes mellitus

Hypertension

Congestive heart failure

Ischemic heart disease

Lung disease

Chronic kidney disease

Immunosuppression

\section{Symptoms}

\begin{tabular}{lcccc} 
Any & $93(83.8)$ & $68(87.2)$ & $20(74.1)$ & 0.13 \\
Fever $^{\mathrm{b}}$ & $54(48.6)$ & $45(57.7)$ & $7(25.9)$ & $\mathbf{0 . 0 0 4}$ \\
\hline Chills $^{\mathrm{b}}$ & $37(33.3)$ & $31(39.7)$ & $5(18.5)$ & $\mathbf{0 . 0 4 5}$ \\
\hline Cough & $69(62.2)$ & $51(65.4)$ & $14(51.9)$ & 0.21 \\
\hline Dyspnea & $45(40.5)$ & $37(47.4)$ & $7(25.9)$ & 0.051 \\
\hline Sore throat & $37(33.3)$ & $21(26.9)$ & $14(51.9)$ & $\mathbf{0 . 0 1 8}$ \\
\hline Headache & $36(32.4)$ & $25(32.1)$ & $8(29.6)$ & 0.81 \\
\hline Myalgia & $47(42.3)$ & $35(44.9)$ & $10(37.0)$ & 0.48 \\
\hline Abdominal pain & $18(16.2)$ & $13(16.7)$ & $3(11.1)$ & 0.76 \\
\hline Nausea and vomiting & $23(20.7)$ & $18(23.1)$ & $3(11.1)$ & 0.18 \\
\hline Diarrhea & $35(31.5)$ & $26(33.3)$ & $6(22.2)$ & 0.28 \\
\hline Loss of smell or taste & $39(35.1)$ & $28(35.9)$ & $6(22.2)$ & 0.19 \\
\hline Treatment & & & & $1(3.7)$ \\
\hline Oxygen support & $15(13.5)$ & $14(17.9)$ & $1(3.7)$ & 0.11 \\
\hline Experimental SARS-CoV-2 directed therapy & $18(16.2)$ & $17(21.8)$ & & $\mathbf{0 . 0 3 7}$ \\
\hline
\end{tabular}

a 6 out of 111 patients examined had intermediate serology

$76(68.5)$

35 (31.5)

$41(36.9)$

$17(15.3)$

7 (6.3)

$14(12.6)$

$1(0.9)$

$6(5.4)$

5 (4.5)

2 (1.8)

3 (2.7)

57 (73.1)

21 (26.9)

28 (35.9)

12 (15.4)

6 (7.7)

9 (11.5)

1 (1.3)

4 (5.1)

2 (2.6)

2 (2.6)

3 (3.8)

18 (66.7)

9 (33.3)

11 (40.7)

0.65

4 (14.8)

1 (3.7)

5 (18.5)

$0(0.0)$

2 (7.4)

2 (7.4)

$0(0.0)$

$0(0.0)$

0.57 
${ }^{\mathrm{b}}$ Independent correlates as revealed by multivariate analysis

Abbreviations: SARS-CoV-2, severe acute respiratory syndrome coronavirus 2 .

The most common presenting symptoms noted in the entire cohort were cough (62.2\%), fever (48.6\%) and dyspnea (40.5\%) (Table 1). IgG-positive patients had significantly higher rates (compared to IgG negative patients) of fever ( $57.7 \%$ vs. $25.9 \%$, $p<0.0001)$, chills $(39.7 \%$ vs. $18.5 \%, p=0.04)$ and dyspnea $(47.4 \%$ vs. $25.9 \%, p=0.047)$ at presentation. Fourteen of the 15 patients who required oxygen support were positive for SARS-CoV-2 IgG, although the difference between the IgG-positive and IgG negative patients did not reach statistical significance $(17.9 \%$ vs. $3.7 \%, p=0.11)$. A significantly higher proportion of IgG positive patients had received SARS-CoV-2 experimental therapies (i.e., hydroxychloroquine, azithromycin, lopinavir/ritonavir) compared to IgG negative patients $(21.8 \%$ vs $3.7 \%, p=0.029)$.

Nasopharyngeal viral RNA was still detected in $23.8 \%$ of the patients at the time of serum sampling. While the detection rate of viral RNA appeared to be somewhat lower among IgG-positive patients (20.5\%) compared with IgG negative patients (33.3\%), this difference did not reach statistical significance $(p=0.18)$.

Comparing additional laboratory parameters, we found that median ferritin levels were higher in the IgG positive group (129.2 vs. $78.9 \mathrm{mg} / \mathrm{dL}, \mathrm{p}=0.039$ ), whereas white blood cell and platelet counts, and CRP levels did not differ between the two groups.

A multivariate analysis identified the presence of fever (OR 5.13; 95\% Cl $1.53-17.45 ; p=0.008$ ), chills (OR $6.82 ; 95 \% \mathrm{Cl} 1.60-29.08 ; \mathrm{p}=0.009$ ), and the time-from-diagnosis (OR 1.18; 95\% $\mathrm{Cl} 1.06-1.32 ; \mathrm{p}=0.004$ ) as independent predictors of the presence of SARS-CoV-2 IgG within the first month from diagnosis.

\section{Semi-quantitative analysis of $\lg \underline{G}$ levels}

A wide ( $>1 \mathrm{log}$ ) IgG level range was identified in $\lg G$ positive patients. Dividing the $\lg G$ positive patients into subgroups with high- and low-antibody levels (with a cutoff set at a ratio of 5.0; see Patients and Methods), we found that patients with high IgG levels were significantly older [51.0 \pm 13.6 vs. $37.4 \pm 18.7$ years, $p<0.001]$. Notably, both ferritin and CRP levels were higher in the high-lgG group with the latter showing a statistically significant difference (median ferritin levels 165.1 vs. $98.1 \mathrm{mg} / \mathrm{dL}, \mathrm{p}=0.14$; median CRP levels 0.36 vs. $0.07 \mathrm{mg} / \mathrm{dL}, \mathrm{p}=0.001$ ). There were no significant differences between the two subgroups with regard to the time post diagnosis, demographic and clinical parameters, and the presence of viral RNA in concurrent nasopharyngeal swab samples.

\section{Discussion}

There is a need to better define the patterns and clinical correlates of antibody response to SARS-CoV-2. By now, several case series and studies of antibody response have been reported in COVID-19 patients, 
focusing primarily on the performance of the different antibody detection assays examined (10-13), and on the analysis of the kinetics of specific antibody response against the viral $\mathrm{S}$ and $\mathrm{N}$ proteins (10).

Here we determined the temporal pattern of SARS-CoV-2 anti-S1 IgG detection in 111 COVID-19 patients who had presented with mild disease, and further identified clinical and laboratory features associated with early antibody response - during the first month from the diagnosis.

While a considerable variability in the timing of antibody response was found between individual patients in this cohort, general antibody detection rates steadily increased from $47.1 \%$ to $93.8 \%$ between the first and the fourth week, with the highest serological shift (60.9\% to $84.4 \%)$ noticed between the second and third week from diagnosis. This observed temporal pattern is overall in agreement with previous reports (14-17), although the use of diverse antibody detection assays (i.e, in-house and commercial ELISA assays, lateral flow immunoassays, pseudotyped-lentiviral-vector-based neutralization assay) along with the relation of antibody detection to the time of symptoms onset (versus the time of diagnosis), and the inclusion of patients with varying disease severity, make it difficult to directly compare the findings in the different studies.

Importantly, we found that in patients with mild COVID-19, the presence of detectable antibodies within a month from diagnosis correlated with more symptomatic disease at presentation. In particular, antibodypositive patients manifested higher rates of fever, chills, and dyspnea (the latter of borderline significance) (Table 1), with a higher rate of experimental SARS-CoV-2-directed therapies (probably reflecting the more profound presentation). Additional symptoms, including myalgia, cough, gastrointestinal symptoms, and loss of taste (with the exception of sore throat), as well as the requirement for oxygen support, were also more common, albeit not reaching statistical significance, in the antibody-positive group. In fact, apart from the time-from-diagnosis, the presence of fever and chills independently correlated with the development of SARS-CoV-2 IgG, as revealed by multivariate analysis. The association of more profound inflammatory-related symptoms with early antibody response could reflect the effect of a higher viral load and/or a more marked innate immune activation on antibody formation. The latter mechanistic notion is supported The recent finding that asymptomatic patients with SARS-CoV-2 infection have reduced inflammatory response and antibody levels relative to symptomatic patients despite longer duration of viral shedding (18). By analogy, early activation of innate immunity is known to translate into higher antibody responses following both infections and vaccinations (19). The correlation between the immune activation state and the extent of antibody response is further suggested by our findings that IgG positive patients had higher ferritin levels $(p=0.039)$, and that higher antibody levels further correlated with higher CRP levels $(p=0.001)$.

While we did not identify a correlation between age and early development of antibodies per se, an older age was found to be associated with higher antibody levels, in agreement with the higher levels of neutralizing antibody titers reported in elderly and middle-age patients compared to young adult patients from China (15). Future studies should explore the underlying mechanism of the age-related enhancement of antibody response to the SARS-CoV-2, and whether it could be related to an augmented 
innate immune response or to the recently reported presence of pre-existing immunity to the virus in older individuals (20).

To our knowledge, this is the first study describing the inflammation-related clinical correlates of early antibody response in patients with mild COVID-19. Our study has several limitations. First, we used a single S1-protein specific ELISA assay, which does not reflect the full array of anti SARS-CoV-2 antibody formation and the functional neutralizing activity (6). Additionally, we did not address the individual kinetics of antibody response, as patients were tested at a single time point within one month from the time of diagnosis (which also differs to a variable extent from the time of disease onset), rather than longitudinally. The inclusion of patients residing in isolation-hotels may have created a bias towards those with continued viral detection. While we showed that neither the presence nor the levels of antibodies correlated with clearance of viral RNA, this finding should be interpreted with caution with regard to its implication for immune protection, in the absence of antibody neutralization or sample infectivity data. Finally, we were unable to define disease severity according to accepted definitions (such as the national early warning score 2 ) due to a lack of information regarding patients' clinical presentation. However, the low rate of oxygen support required and COVID-19 directed therapy and the lack of ICU admissions, support the result as representing the serology patterns of mild COVID-19 cases. Despite these limitations, patients with mild disease constitute the majority of COVID-19 patients, hence focusing on a large cohort of these patients, the use of an ELISA assay which has been shown to be comparable with other major commercially-available assays (https://www.fda.gov/medicaldevices/emergency-situations-medical-devices/eua-authorized-serology-test-performance; $(21,22)$ ), along with the recently demonstrated lower antibody response in asymptomatic patients (18), support the generalizability of our findings.

\section{Conclusions}

Our findings, demonstrating the patient-to-patient variability and the general temporal pattern of SARSCoV-2 IgG detection show that in patients with mild COVID 19, fever and chills along with basic inflammation-related markers, positively correlate with enhanced IgG response during the early period post diagnosis. Older age and higher CRP levels are associated with enhanced antibodies response among IgG positive patients. These findings, along with future analysis of additional innate immune activation markers, long-term follow-up, and functional antibody studies, could contribute to better prediction and understating of the immune response against SARS-CoV-2, and inform therapeutic donor plasma selection.

\section{List Of Abbreviations}

SARS-CoV-2, severe acute respiratory syndrome coronavirus 2; COVID-19, coronavirus disease 19; ARDS, acute respiratory distress syndrome; RT, reverse transcriptase; PCR, polymerase chain reaction; $\mathrm{CRP}, \mathrm{C}$ reactive protein; ELISA, enzyme-linked immunosorbent assay; 


\section{Declarations}

Ethics Approval and Consent to Participate: The study was approved by the institutional Helsinki committee. Patient included after providing informed consent.

Consent for Publication: Not applicable

Data Availability: The datasets generated and/or analysed during the current study are not publicly available, due to patient discretion, but are available from the corresponding author on reasonable request.

Competing Interests: The authors have no conflict of interest to declare regarding this manuscript.

Funding: No external funding was used to conduct the presented research

Authors' Contribution: All authors participated in conducting the presented study. The manuscript was prepared by $\mathrm{AK}, \mathrm{AK}, \mathrm{DW}$ and $\mathrm{YI}$ and was revised and edited by all the other authors.

Acknowledgments: We thank Aliza Lifshitz and Sarit Doron Mizrahi for technical assistance.

\section{References}

1. Huang C, Wang Y, Li X, Ren L, Zhao J, Hu Y, et al. Clinical features of patients infected with 2019 novel coronavirus in Wuhan, China. Lancet. 2020;395(10223):497-506.

2. Wu Z, McGoogan JM. Characteristics of and Important Lessons From the Coronavirus Disease 2019 (COVID-19) Outbreak in China: Summary of a Report of 72314 Cases From the Chinese Center for Disease Control and Prevention. Jama. 2020.

3. Grasselli G, Zangrillo A, Zanella A, Antonelli M, Cabrini L, Castelli A, et al. Baseline Characteristics and Outcomes of 1591 Patients Infected With SARS-CoV-2 Admitted to ICUs of the Lombardy Region, Italy. Jama. 2020.

4. Cummings MJ, Baldwin MR, Abrams D, Jacobson SD, Meyer BJ, Balough EM, et al. Epidemiology, clinical course, and outcomes of critically ill adults with COVID-19 in New York City: a prospective cohort study. Lancet. 2020;395(10239):1763-70.

5. Li T. Diagnosis and clinical management of severe acute respiratory syndrome Coronavirus 2 (SARSCoV-2) infection: an operational recommendation of Peking Union Medical College Hospital (V2.0). Emerg Microbes Infect. 2020;9(1):582-5.

6. Robbiani DF, Gaebler C, Muecksch F, Lorenzi JCC, Wang Z, Cho A, et al. Convergent antibody responses to SARS-CoV-2 in convalescent individuals. Nature. 2020.

7. Woloshin S, Patel N, Kesselheim AS. False Negative Tests for SARS-CoV-2 Infection - Challenges and Implications. N Engl J Med. 2020. 
8. Kucirka LM, Lauer SA, Laeyendecker O, Boon D, Lessler J. Variation in False-Negative Rate of Reverse Transcriptase Polymerase Chain Reaction-Based SARS-CoV-2 Tests by Time Since Exposure. Ann Intern Med. 2020.

9. Shen C, Wang Z, Zhao F, Yang Y, Li J, Yuan J, et al. Treatment of 5 Critically III Patients With COVID19 With Convalescent Plasma. Jama. 2020.

10. Li Z, Yi Y, Luo X, Xiong N, Liu Y, Li S, et al. Development and clinical application of a rapid IgM-lgG combined antibody test for SARS-CoV-2 infection diagnosis. J Med Virol. 2020.

11. Padoan A, Cosma C, Sciacovelli L, Faggian D, Plebani M. Analytical performances of a chemiluminescence immunoassay for SARS-CoV-2 IgM/lgG and antibody kinetics. Clin Chem Lab Med. 2020.

12. Okba NMA, Müller MA, Li W, Wang C, GeurtsvanKessel CH, Corman VM, et al. Severe Acute Respiratory Syndrome Coronavirus 2-Specific Antibody Responses in Coronavirus Disease 2019 Patients. Emerg Infect Dis. 2020;26(7).

13. Spicuzza L, Montineri A, Manuele R, Crimi C, Pistorio MP, Campisi R, et al. Reliability and usefulness of a rapid IgM-lgG antibody test for the diagnosis of SARS-CoV-2 infection: A preliminary report. J Infect. 2020.

14. Wölfel R, Corman VM, Guggemos W, Seilmaier M, Zange S, Müller MA, et al. Virological assessment of hospitalized patients with COVID-2019. Nature. 2020;581(7809):465-9.

15. Wu F, Wang A, Liu M, Wang Q, Chen J, Xia S, et al. Neutralizing antibody responses to SARS-CoV-2 in a COVID-19 recovered patient cohort and their implications. medRxiv. 2020:2020.03.30.20047365.

16. Ni L, Ye F, Cheng ML, Feng Y, Deng YQ, Zhao H, et al. Detection of SARS-CoV-2-Specific Humoral and Cellular Immunity in COVID-19 Convalescent Individuals. Immunity. 2020.

17. Jääskeläinen AJ, Kekäläinen E, Kallio-Kokko H, Mannonen L, Kortela E, Vapalahti O, et al. Evaluation of commercial and automated SARS-CoV-2 lgG and lgA ELISAs using coronavirus disease (COVID19) patient samples. Euro Surveill. 2020;25(18).

18. Long QX, Tang XJ, Shi QL, Li Q, Deng HJ, Yuan J, et al. Clinical and immunological assessment of asymptomatic SARS-CoV-2 infections. Nat Med. 2020.

19. Dowling DJ, Levy O. Pediatric Vaccine Adjuvants: Components of the Modern Vaccinologist's Toolbox. Pediatr Infect Dis J. 2015;34(12):1395-8.

20. Braun J, Loyal L, Frentsch M, Wendisch D, Georg P, Kurth F, et al. Presence of SARS-CoV-2 reactive T cells in COVID-19 patients and healthy donors. medRxiv. 2020:2020.04.17.20061440.

21. Kohmer N, Westhaus S, Rühl C, Ciesek S, Rabenau HF. Clinical performance of different SARS-CoV-2 IgG antibody tests. J Med Virol. 2020.

22. Geurtsvankessel C, Okba N, Iglói DZ, Embregts C, Laksono B, Leijten L, et al. Towards the next phase: evaluation of serological assays for diagnostics and exposure assessment2020.

\section{Figures}




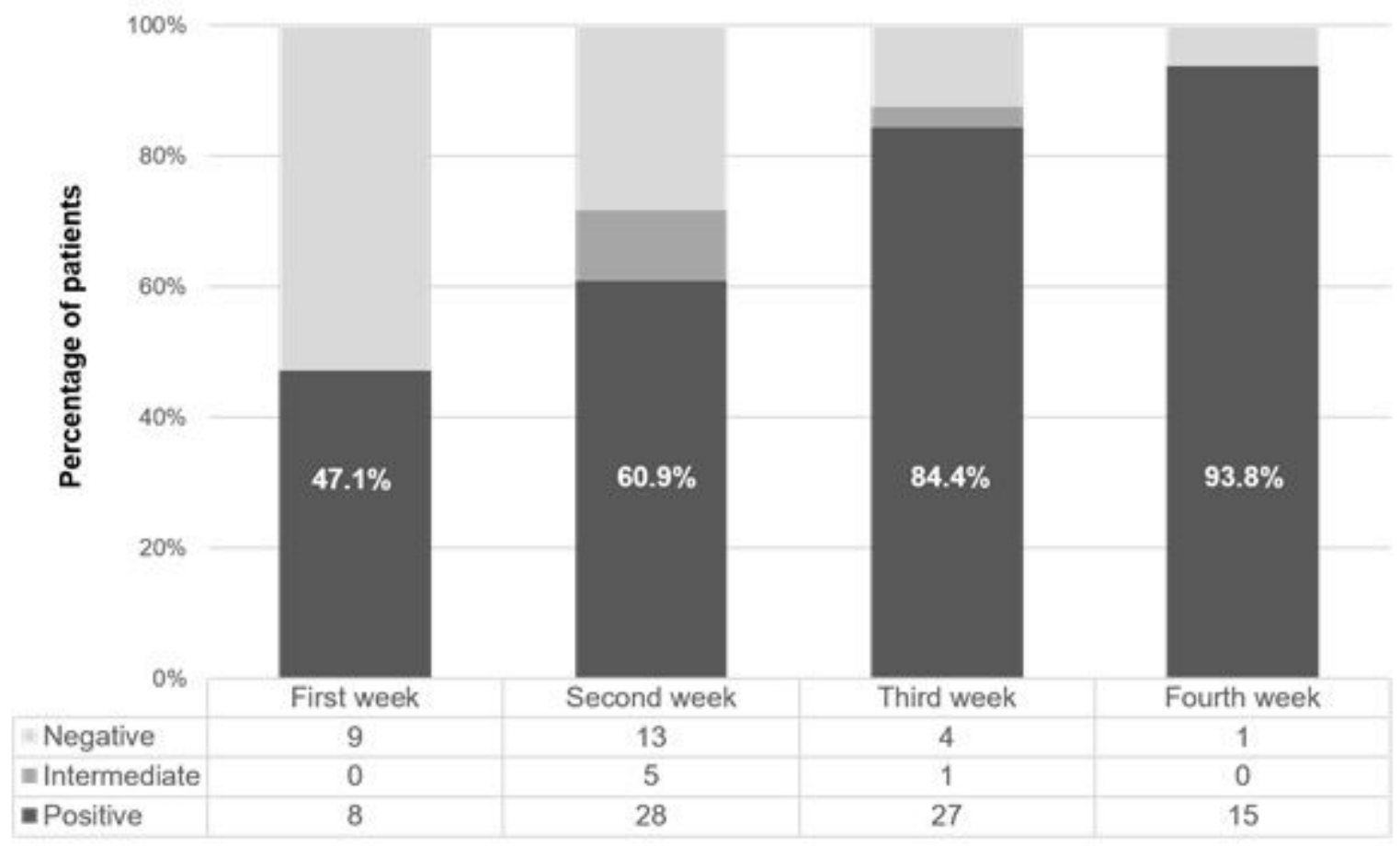

Figure 1

Temporal pattern of SARS-COV-2 IgG detection. Time from diagnosis is presented by weeks. 